\title{
Tratamento de varizes com laser endovenoso: estudo prospectivo com seguimento de 39 meses
}

\author{
Treatment of varicose veins with endovenous laser: a prospective 39-month follow-up study
}

\author{
Luiz M arcelo Aiello Viarengo ${ }^{1}$, G uilherme Vieira M eirelles ${ }^{2}$, João Potério Filho ${ }^{3}$
}

\begin{abstract}
Resumo
O bjetivo: Avaliar os resultados da terapêutica endovenosa com laser no tratamento de varizes primárias.

Pacientes e método: No período compreendido entre julho de 2001 e setembro de 2004 (39 meses), 253 pacientes (417 membros) foram tratados ambulatorialmente com laser de diodo de 810 e 980-nm, com energia liberada endovenosamenteatravés de fibra óptica introduzida por punção guiada por eco-D oppler. Foi utilizada anestesia por infiltração intumescente perivasal com solução de lidocaína a 0,2\% (50-150 ml). A potência e duração do pulso foram determinadas pelo diâmetro da veia. 0 s controles foram realizados com ecoD oppler aos 7 dias, 1 mês, 3 meses, 6 meses, 1 ano e, a seguir, anualmente, para avaliar a eficácia do tratamento e os efeitos adversos.

Resultados: A oclusão primária da veia safena magna foi obtida em 405 dos 417 membros $(97,1 \%)$ tratados. Houve reintervenção em 12 casos (2,9\%), com sucesso. 0 tempo médio de observação foi de 18 meses, e, nesse período, a taxa de recidiva global de varizes foi de $7,4 \%$, sendo $6,3 \%$ (26 membros) veias colaterais tributárias da crossa e 1,2\% (cinco membros) com recanalização da safena magna. Todas as recorrências ocorreram entre o terceiro e $012^{\circ}$ mês. O s efeitos indesejáveis mais freqüentes foram: equimoses $(60,6 \%)$; dor suportável durante o procedimento $(16,1 \%)$; hematomas $(5,5 \%)$; flebite em colaterais não-tratadas (3,4\%); hiperpigmentação $(2,9 \%)$; e parestesia transitória (3,4\%). N enhum caso de tromboflebite da safena magna foi observado. $\mathrm{N}$ ão houve nenhum caso de trombose venosa profunda ou embolia pulmonar.

C onclusão: 0 tratamento endovenoso de varizes com laser, conforme descrito, foi eficaz para ocluir a safena magna e seus principais ramos, com efeitos adversos autolimitados e com recorrência inferior a $8 \%$ no período de seguimento.
\end{abstract}

Palavras-chave: Varizes, cirurgia a laser, lasers.

\begin{abstract}
O bjective: To evaluate results of endovenous laser treatment for primary varicose veins.

Patients and method: From July 2001 to September 2004 (39 months), 253 outpatients (417 limbs) were treated with 810 and 980 $\mathrm{nm}$ diode laser energy delivered percutaneously using optical fiber introduced by puncture under ultrasound guidance. Tumescent anesthesia (50-150 $\mathrm{ml}$ of $0.2 \%$ lidocaine) was delivered perivenously. Power and duration of the pulse were determined by vein diameter. Duplex control was performed at 7 days, 1 month, 3 months, 6 months, 1 year and yearly thereafter to assess treatment efficacy and adverse effects.

Results: Primary great saphenous vein occlusion was obtained in 405 of 417 members (97.1\%). Twelve recurrent cases (2.9\%) were successfully treated. M ean follow-up time was 18 months. During this period, global recurrence rate of varicose veins was $7.4 \% ; 6.3 \%$ (26 limbs) related to tributary and collateral veins of the saphenofemoral junction, and $1.2 \%$ (five limbs) with great saphenous vein recanalization. All recurrences occurred between 3 and 12 months. Ecchymosis was the most common adverse effect $(60.6 \%)$. O ther complications were: moderate pain during the procedure $(16.1 \%)$; hematoma (5.5\%), superficial phlebitis of varicose tributaries $(3.4 \%)$, hyperpigmentation $(2.9 \%)$, transient paresthesia $(3.4 \%)$. T here were no cases of great saphenous vein thrombophlebitis, deep vein thrombosis or pulmonary emboli.

Conclusion: Varicose vein treatment with endovenous laser technique was successful in occluding great saphenous vein and its branches, with self-limited adverse effects and recurrence rate lower than $8 \%$ in the follow-up period.
\end{abstract}

Key words: Varicose veins, laser surgery, lasers.

1. Cirurgião vascular, ultra-sonografista vascular. Pós-graduando em Cirurgia, Universidade Estadual de Campinas (UNICAMP), Campinas, SP.

2. Cirurgião vascular. Mestre em Cirurgia e Pós-graduando em Cirurgia, UNICAMP, Campinas, SP.

3. Livre-docente, professor adjunto da disciplina de Cirurgia Vascular, UNICAMP, Campinas, SP.

O trabalho foi desenvolvido no Instituto Meirelles-Viarengo - Centro de Estudo, Diagnóstico e Tratamento de Doenças da Circulação e no Instituto Cardiológico de Campinas - Hospital e Pronto-Socorro do Coração. O trabalho foi apresentado na sessão de temas-livres especiais (TLE-009) do 36ำ Congresso Brasileiro de Angiologia e Cirurgia Vascular, realizado em Porto Alegre, RS, de 4 a 8 de setembro de 2005 (publicado em J Vasc Bras. 2005;4(3 Suppl. 1).

Artigo submetido em 03.05.06, aceito em 06.09.06. 


\section{Introdução}

As varizes constituem a mais comum de todas as alterações vasculares. Estudos epidemiológicos realizados por $M$ affei et al. mostram uma prevalência na população geral, em indivíduos com mais de 15 anos, de $35,5 \%$, sendo $15,8 \%$ de casos mais graves ${ }^{1}$.

0 tratamento cirúrgico padrão para a maioria dos pacientes nos estágios clínicos C 2 a C 6, com comprovada incompetência da junção safeno-femoral (JSF) e refluxo ao longo da veia safena magna (VSM), é a ligadura de todas as tributárias da crossa, com ligadura daJSF, fleboextração daVSM eflebectomia por avulsão das colaterais, ramos e perfurantes 2 .

D eacordo com a opinião prevalente, baixastaxas de recorrência deveriam ser esperadas se o refluxo da JSF foi adequadamente controlado e se a VSM foi ressecada com interrupção de todas as tributárias proximais ${ }^{3,4}$.

Entretanto, a cirurgia de varizes, embora considerada um procedimento menor e seguro, realizado há cerca de 100 anos com poucas variações e inovações técnicas e com resultados conhecidos, é associada a uma taxa não-desprezível de morbidade cirúrgica, insatisfação do paciente e recorrências 5 . Tal fato nos estimula a investigar eavaliar novos métodos de tratamento, na busca de alternativas que possam acrescentar benefícios ao paciente, ao sistema de saúde e à sociedade.

O objetivo deste estudo foi avaliar, prospectivamente, os resultados (eficácia e efeitos adversos) da terapêutica com laser en dovenoso (EVL) no tratamento da doença varicosa.

\section{Pacientes e método \\ Pacientes}

No período compreendido entre junho de 2001 e setembro de 2004 (39 meses), 253 pacientes, com 417 membroscom insuficiênciavenosa crônica (IVC) e classificação CEAP C 2-6, Ep, As, Pr, foram submetidos ao tratamento cirúrgico de varizes com EVL no Instituto M eirelles-Viarengo - Centro de Estudo, $D$ iagnóstico e T ratamento de D oenças da Circulação, em Campinas (SP). A amostra não foi consecutiva nem aleatória.

Os pacientes foram previamente triados para 0 propósito do estudo. 0 sindivíduos selecionadospara o tratamento endovenoso devarizes com laser eram todos portadores de IVC de origem primária, com ou sem lesões tróficas e que concordaram com o tratamento.

Aqueles que apresentavam doença venosa aguda trombose venosa profunda (TVP), tromboflebite -, síndrome pós-trombótica, trombofilias ou estados de hipercoagulabilidade, insuficiência arterial, gestantes, incapacidade para deambulação, doenças sistêmicas descompensadas e estado geral precário não foram elegíveis para o tratamento.

Este estudo foi submetido e aprovado pela comissão de ética sob parecer 01/2001. O s pacientes foram informados quanto aos objetivos da pesquisa, riscos e resultados esperados. A pós assistirem aula informativa, todos que concordaram foram tratados, após consentimento informado escrito e de acordo com os preceitos éticos da D eclaração de $\mathrm{H}$ elsinki.

T odos os pacientes foram avaliados pré-operatoriamentecom eco-D oppler colorido (AT L H D I 3000 ou Toshiba N emio), para demonstrar a incompetência da VSM , daJSF e da veia safena parva (VSP). Foi considerado refluxo significativo aquele com duração de pelo menos 2 segundos, com paciente em ortostatismo, durante manobra de Valsalva ou com compressão/ descompressão da panturrilha. T odos os exames foram realizados pelo mesmo examinador e com as mesmas condições laboratoriais. As características dos 253 pacientes (417 membros) submetidos ao tratamento com 0 $E V L$, de acordo com a classificação clínica, etiológica, anatômicae fisiopatológica(CEAP), estão expressasna Tabela 1.

\section{Procedimento}

Para o tratamento, foi utilizado equipamento de laser de diodo com comprimento de onda de $810 \mathrm{~nm}$ e 980 nm, potência óptica de 15 W emodo de operação pulsado. 0 protocolo atual mente empregado foi modificado e adaptado dos trabalhos de $\mathrm{N}$ avarro et al. ${ }^{6}$.

I mediatamenteantes do tratamento, os pacientes foram submetidos a um mapeamento com eco-D oppler colorido, em ortostatismo, para determinar 0 diâmetro da veia a ser tratada, sendo seu valor anotado no próprio membro, a cada $10 \mathrm{~cm}$ a partir daJ SF. A quantidade de energia (E) liberada por pulso, em cada segmento, foi de 3 joules/mm de diâmetro. $0 \mathrm{~s}$ acertosnos parâmetros do equi pamento para se obter a quantidade de energia cal culada, por pulso, foram realizados ajustando o tempo de duração do pulso ( $T$ ) e a potência $(P)$, onde $E=T \times P$. A velocidade de 
retirada da fibra foi tal que foram liberados tantos pulsos quantos necessários para garantir a entrega de um mínimo de 80 joules por centímetro linear 7,8 .

Tabela 1 - C aracterísticas dos 253 pacientes $(417$ membros) tratados com EVL, de acordo com a classificação CEAP

\begin{tabular}{lc}
\hline & $\begin{array}{c}\text { Número de } \\
\text { membros (\%) }\end{array}$ \\
\hline Estágio clínico & \\
C2 - veias varicosas com $>4 \mathrm{~mm}$ & $47(11,2 \%)$ \\
C3 - edema & $48(11,2 \%)$ \\
C 4 - alterações tróficas & $161(38,8 \%)$ \\
C5 - úlcera cicatrizada & $124(29,9 \%)$ \\
C6 - úlcera ativa & $37(8,9 \%)$ \\
Etiologia & \\
Primária & $417(100 \%)$ \\
Secundária & 0 \\
Anatomia & \\
Veias superficiais & $417(100 \%)$ \\
Veias profundas & 0 \\
Fisiopatologia & \\
Refluxo & $417(100 \%)$ \\
O bstrução & 0 \\
\hline
\end{tabular}

$\mathrm{EVL}=$ laser endovenoso; CEAP = classificação clínica, etiológica, anatômica e fisiopatológica.

T odosospacientesreceberam 15 mg deM idazolam por via oral, 30 minutos antes do procedimento.

A punção daVSM ou VSP foi realizadana extremidade distal do refluxo orientado pelo eco-Doppler, utilizando uma agulha $16 \mathrm{G}$ ou $18 \mathrm{G}$. Retirado o mandril, a fibra óptica foi introduzida diretamente na veia, com manobras suaves, sem auxílio de cateter-guia. Q uando ocorreu qual quer resistência ou dificuldadena progressão, a fibra óptica foi retirada, passando-se um fio-guia) 0,035-in avançado, por meio decontrolecom eco-D oppler, atéa) SF. U m cateter $5 F$ (I nfiniti, $100 \mathrm{~cm}$, $0,97 \mathrm{~mm}$, vertebral, Cordis) foi passado sobre o fio-guia elocado a cerca de $1 \mathrm{~cm}$ da JSF 0 fio-guiafoi removido e substituído pela fibra óptica de 600 micras, com diâmetro externo de $1 \mathrm{~mm}$, introduzida até exteriorizar-se cerca de 6 a $8 \mathrm{~mm}$. 0 posicionamento final da fibra, imediatamente distal à inserção da veia epigástrica superficial, foi realizado sob estrito controle com
eco-D oppler. A anestesia local por infiltração intumescente, com 50 a $150 \mathrm{ml}$ de lidocaína a 0,2\%, foi realizada sob controle com eco-D oppler, ao longo de toda a extensão do vaso a ser tratado. A energia laser foi liberada em modo pulsado, com potência e duração do pulso variando de acordo com o diâmetro do vaso e retração da fibra óptica sendo executada no intervalo entreospulsos. Esseciclo foi repetido atéuma distância de $1 \mathrm{~cm}$ do sítio de punção. Subseqüentemente, 0 cateter foi retirado, e realizou-se o tratamento de colaterais, ramos e perfurantes, com EVL, através de múltiplas punções ou deminiflebectomia clássica. A seguir, o pacienterecebeu uma compressão elástica classell (20 a $30 \mathrm{mmH} \mathrm{g}$ ) em toda a extensão do membro e foi liberado para deambulação após 24 horas. A compressão elástica foi mantida por 30 dias.

Durante o procedimento, os pacientes foram questionados quanto à dor, e suas respostas contemplavam apenas três alternativas: sem dor, suportável e intolerável.

\section{Controle}

T odos os pacientes foram submetidos a exame com eco-D oppler colorido, antes, durantee apóso EV L (aos 7 dias, 30 dias, trimestralmenteaté completar o primeiro ano e semestralmente a partir do segundo ano). 0 sucesso imediato do tratamento foi demonstrado pela identificação da VSM ou da V SP completamente ocluída e sem nenhum sinal de fluxo no interior do vaso. 0 insucesso foi definido por presença de segmento maior ou igual a $10 \mathrm{~cm}$ de extensão, não-ocluído ou parcialmenteocluído. 0 tempo deseguimento médio foi de 18 meses, variando de 30 dias a 39 meses.

\section{Análise estatística}

As variáveis categóricas foram apresentadas em tabelas de contingência, contendo valores absolutos e relativos. O s valores numéricos foram analisados com estatística descritiva.

\section{Resultados}

0 endolaser foi utilizado para tratar 253 pacientes (417 membros) na faixa etária de 18 a 82 anos, com idade média de 53,8 anos e insuficiência da VSM, associada ou não a insuficiência da VSP, colaterais e perfurantes (T abela 2). 0 diâmetro da VSM variou de 5 a 22,4 mm, medidos $3 \mathrm{~cm}$ abaixo da JSF, e o da VSP 
variou de 4 a 9,5 mm, medidos $3 \mathrm{~cm}$ abaixo da prega poplítea. Do total dos pacientes, 182 eram do sexo feminino $(71,9 \%)$, e 71 , do sexo masculino $(28,1 \%)$. Em 164 pacientes (64\%) , 0 acometimento era bilateral, e, em 89 pacientes (36\%), unilateral; 118 mulheres $(63,7 \%)$ e 46 homens $(64,8 \%)$ apresentavam acometimento bilateral ( $\mathrm{T}$ abela 3 ).

T abela 2 - D istribuição dos segmentos venosos tratados

\begin{tabular}{lc}
\hline Segmento venoso & $\begin{array}{c}\text { Número de } \\
\text { membros }(\%)\end{array}$ \\
\hline VSM inteira & $288(68,8 \%)$ \\
VSM supragenicular & $129(31,2 \%)$ \\
VSP & $75(18 \%)$ \\
O utras veias & $278(67 \%)$ \\
\hline
\end{tabular}

VSM = veia safena magna; $\mathrm{VSP}=$ veia safena parva

Foram tratados 237 membrosesquerdos $(57,1 \%)$ e 178 membros direitos (42,9\%). Em 103 membros $(24,8 \%)$, houve dificuldade na punção da veia ou na progressão da fibra óptica, sendo que, em 46 membros $(11,08 \%)$, foram necessárias mais de duas punções em sítios diferentes e, em 57 membros (13,7\%), dissecção por miniflebectomia e mais de duas punções em sítios diferentes para completar o tratamento.
A taxa de sucesso imediato, com oclusão de tronculares(VSM eV SP) foi de 97,1\% (405 membros), sendo necessário reintervir em 12 membros (2,9\%) com a mesma técnica, com uma taxa de sucesso de $100 \%$ na segundaintervenção. D os 278 membrosqueapresentavam varicosidades em outras veias (colaterais, ramos, etc.) e que foram tratados com endolaser, a taxa de sucesso com oclusão das colaterais foi de 90,3\% (251 membros), sendo necessário reintervir em 9,7\% (27 membros). $N$ esses casos, a reintervenção foi realizada com miniflebectomia ambulatorial.

Após o tratamento com endolaser, 369 membros $(88,5 \%)$ apresentaram correção do refluxo na JSF, demonstrada por estudo não-invasivo com eco-D oppler colorido. Entretanto, 48 membros $(11,5 \%)$ permaneceram com refluxo ostial, ainda que a obliteração da VSM tenha sido efetiva.

Entre os 369 membros que tiveram o refluxo na JSF corrigido após o EVL, 19 membros (5,1\%) apresentaram recorrência de varizes na coxa, e, em cinco membros $(1,4 \%)$, foi observada recanalização parcial da VSM , ocorrendo entre 6 e 12 meses após 0 tratamento. A taxa de recidiva de varizes nos membros com correção do refluxo ostial foi de $6,5 \%$. Entre os 48 membros que permaneceram com refluxo ostial após o tratamento com laser, houve recorrência de varizes na coxa, a partir da crossa da V SM , em sete membros (14,6\%), entre 3 e 9 meses após 0 tratamento. A taxa de recidiva global de varizes nos 417 membros tratados, com um seguimento médio de 18 meses, variando de 30 dias a 39 meses, foi de 7,4\% (T abela 4).

T abela 3 - D istribuição dos pacientes por sexo

\begin{tabular}{lcccc}
\hline & $\begin{array}{c}\mathbf{N} \text { o de } \\
\text { pacientes (\%) }\end{array}$ & $\begin{array}{c}\mathbf{N} \text { o de } \\
\text { membros (\%) }\end{array}$ & $\begin{array}{c}\mathbf{N} \text { - de membros com } \\
\text { acometimento bilateral (\%) }\end{array}$ & $\begin{array}{c}\text { No de membros com } \\
\text { acometimento unilateral (\%) }\end{array}$ \\
\hline M ulheres & $182(71,9 \%)$ & 300 & $118(63,7 \%)$ & $64(36,3 \%)$ \\
H omens & $71(28,1 \%)$ & 117 & $46(64,8 \%)$ & $25(36,2 \%)$ \\
Total & $253(100 \%)$ & 417 & $164(64 \%)$ & $89(36 \%)$ \\
\hline
\end{tabular}


T abela 4 - T axa de recorrência de varizes pós-EV L

\begin{tabular}{lccc}
\hline $\begin{array}{l}\text { Condição do refluxo } \\
\text { na JSF pós-EVL }\end{array}$ & $\mathbf{n}(\%)$ & $\begin{array}{c}\text { Recorrência de varizes } \\
\text { pós-EVL (\%) }\end{array}$ & $\begin{array}{c}\text { Intervalo de tempo } \\
\text { para recorrência }\end{array}$ \\
\hline Ausente & $369(88,5 \%)$ & $24(6,5 \%)$ & $6-12$ meses \\
Presente & $48(11,5 \%)$ & $7(14,6 \%)$ & $3-9$ meses \\
Total & $417(100 \%)$ & $31(7,4 \%)$ & \\
\hline
\end{tabular}

$\mathrm{EVL}=$ laser endovenoso; JSF = junção safeno-femoral.

\section{Efeitos adversos}

0 procedimento realizado com anestesia local por infiltração intumescente foi muito bem tolerado pela maioria dos pacientes. Entretanto, em 67 casostratados $(16,1 \%)$, os pacientes queixaram-se de dor "suportável", não impedindo ou dificultando a continuidade do procedimento. N ão houve nenhum caso de dor "intolerável". Equimoses foram observadas em 251 mem$\operatorname{bros}(60,2 \%)$, no trajeto da VSM , sendo mais acentuadas no terço médio e distal da coxa, desaparecendo completamente, sem seqüelas, ao final da quarta semana. Em 23 membros (5,5\%), houve a formação de pequeno hematoma na virilha e face medial do terço superior e médio da coxa. Em todos os casos, houve regressão espontânea sem complicações. Flebite foi observada em 14 membros $(3,4 \%)$, sendo que, em todosos casos, as veias acometidas foram colateraisnãotratadas. $\mathrm{N}$ ão houvenenhum caso deflebite com manifestações clínicas nas veias tratadas com EVL. Em 12 membros (2,9\%), ocorreu hiperpigmentação, sendo 11 casos nas áreas sobre as veias com flebite; em um caso, a pigmentação foi no trajeto da V SM . Parestesia transitóriafoi observada em 14 membros(3,4\%), localizada na face medial do terço médio edistal da perna e região inframaleolar medial e/ ou lateral. T odos os casos apresentaram remissão completa espontânea ao final do terceiro mês, sem necessidade de nenhum tratamento adicional. D ois pacientes $(0,5 \%)$ evoluíram com um quadro de neurite severa na face medial da coxa, sendo necessário tratamento com antidepressivos tricíclicos, gabapentina, tiamina, piridoxina e cianocobalamina. U m caso foi resolvido com 4 meses de tratamento, e o outro, com 8 meses. Em um paciente $(0,24 \%)$ com classificação clínica C6, ocorreu uma complicação infecciosa no sítio de punção, sendo necessária antibioticoterapia por 10 dias. Em um caso $(0,24 \%)$, houve queimadura de pele na face medial do terço médio da perna, com formação de flictena e úlcera, completamenteresolvida ao final do terceiro mês, deixando uma pequena seqüela cicatricial. Em cinco casos $(1,2 \%)$, foi observada extensão do trombo da VSM para a veia femoral comum, com resolução espontânea completa ao final do primeiro mês. Todos os pacientes, com exceção dos dois casos com neurite, estavam satisfeitos com a decisão de serem submetidos ao tratamento com o EVL ecom os resultados subseqüentes. N enhum caso de tromboflebite da VSM ou VSP foi observado. N ão houvenenhum caso deT VP ou embolia pulmonar (EP) (T abela 5).

\section{D iscussão}

0 EVL para tratamento de varizes tronculares com comprovado refluxo na JSF e V SM surge como uma alternativa bastante atrativa à ligadura alta da JSF efleboextração da V SM , sobretudo por permitir que 0 paciente mantenha suas atividades habituais sem limitações significativas. 0 laser oclui a VSM sem afetar as tributárias no nível da JSF, fato que torna o método muito controverso, considerando que geralmente é aceito, entre os especialistas, que uma das causas mais freqüentes de recorrência de varizes é a falta de controle adequado do refluxo na JSF e das tributárias da crossa 3,9,10.

Com base nesses princípios teóricos, que são quase dogmas no tratamento de varizes tronculares, podería- 
T abela 5 - Efeitos adversos

\begin{tabular}{lc}
\hline Efeito adverso & Número de membros (\%) \\
\hline D or "suportável" durante o procedimento & $67(16,1 \%)$ \\
E quimoses & $251(60,6 \%)$ \\
H ematomas & $23(5,5 \%)$ \\
Flebite (colaterais) & $14(3,4 \%)$ \\
H iperpigmentação & $12(2,9 \%)$ \\
Parestesia transitória & $14(3,4 \%)$ \\
N eurite (face medial da coxa) & $2(0,5 \%)$ \\
Infecção & $1(0,2 \%)$ \\
Q ueimadura de pele & $1(0,2 \%)$ \\
Extensão do trombo para a VFC & $5(1,2 \%)$ \\
Tromboflebite VSM /V SP & 0 \\
Trombose venosa profunda & 0 \\
Embolia pulmonar & 0 \\
\hline
\end{tabular}

$\mathrm{VFC}=$ veia femoral comum; $\mathrm{VSM}=$ veia safena magna $\mathrm{VSP}=$ veia safena parva.

mos prever o insucesso do EVL, que, sob um olhar menos atento e revestido de uma crítica defensiva, poderia atribuir-lhe apenas o glamour que desperta junto à população leiga, além de agregar custos com resultados duvidosos.

Por outro lado, não podemos afirmar que a cirurgia, baseada nesses preceitos, tenha resolvido o problema das varizes esuas recorrências, considerando que, na literatura, essa recorrência podeser tão al ta quanto 40\% ou mais, em 5 anos $^{5}$. Certamente, essa questão não está encerrada. Ainda nos falta um entendimento mais completo das causas que determinam níveis tão el evados de recorrência após uma cirurgia realizada de acordo com todos os preceitos técnicos.

D epois do estudo de G lass, em 1987, evidências têm sido acumuladas para sustentar quea neovascularização, queéconsiderada como uma exten são do processo de angiogênese que ocorre na reparação da ferida cirúrgica na virilha, é a maior fonte de novos canais, reconectando as veias superficiais com a veia femoral comum ao redor da JSF $11-13$.

$M$ aisrecentemente, Fischer, em um estudo com um seguimento médio de 34 anos, para verificar a recorrência tardia do refluxo na JSF após a ligadura alta desta junção, com ligadura de todas as tributárias da crossa e fleboextração da VSM na coxa, demonstrou uma incidência de $60 \%$ de recorrência de refluxo na JSF, sendo que, em $71 \%$ dos casos, o refluxo se originava no sítio de ligadura da antiga JSF, e, em $29 \%$, a origem era perijuncional ${ }^{14-16 .}$

0 tratamento cirúrgico de varizes não é uma questão encerrada, e os dogmas não se constituem em verdades absolutas. As evidências sugerem que a abordagem cirúrgica, como preconizada até então, pode constituir-se no principal evento desencadeador de fenômenos biológicos responsáveis pelas recorrências de varizes a partir do sítio de ligadura da JSF, sendo, portanto, paradoxalmente contrária aos próprios objetivos da cirurgia.

N o presente estudo, em 369 membros (88,5\%), 0 refluxo da JSF foi corrigido no tratamento inicial, provavelmente por retração da parede venosa secundária ao efeito térmico e desnaturação do colágeno. Entre esses membros, foi observado, no período de seguimento médio de 18 meses (variando de 30 dias a 39 meses), 19 recorrências de varizes na coxa $(5,1 \%)$ e cinco casos $(1,4 \%)$ de recanalização parcial da VSM . Entre os 48 membros $(11,6 \%)$ que permaneceram com refluxo 
ostial após o tratamento com endolaser, houve sete recorrências de varizes $(14,6 \%)$ a partir da crossa da VSM. Todas as recorrências ocorreram entre 3 e 12 meses pós-tratamento ( $T$ abela 4). A póso primeiro ano, até 0 encerramento do estudo, não houve novas recorrências ${ }^{8}$. Comparando a taxa de recorrência de varizes entre o pacientesquetiveram o refluxo naJ SF corrigido e aquel es que permaneceram com o refluxo, não houve diferença com significado estatístico $(P=0,4328)$.

Se compararmosa taxa de recidiva gl obal de varizes nos 417 membros tratados, de 7,4\% no período de segmento médio de 18 meses (variando de 30 dias a 39 meses), com as taxas de recidivas relatadas na literatura para a cirurgia convencional, variando de 26 a $28 \%$ no período de 2 a 5 anos ${ }^{9,17}$, ainda que este não seja um resultado conclusivo, parece-nos favorável ao EVL.

T anto os resultados, com taxa de sucesso imediato de $97,1 \%$, como os efeitos adversos observados são semelhantes aos relatados na literatura ${ }^{6,8,18-27}$, não havendo nenhum caso revestido de maior gravidade. O s casos que mais geraram desconforto para a equipe foram os dois pacientes que cursaram com neuritecom resolução demorada, porém sem nenhuma seqüela (T abelas 5, 6 e 7).
0 maior temor e motivo de críticas ao tratamento endovenoso devarizes tronculares com laser refere-seao risco de TVP e EP, secundário ao processo de oclusão trombótica fototérmica da VSM , com progressão do trombo para a veia femoral comum, em virtude da nãoligadura cirúrgica da JSF. Entretanto, parece-nos que tal temor não encontra sustentação em evidências científicas.

Em um levantamento das complicações tromboembólicas relatadas nas principais séries publicadas na literatura, excluídos os casos que aparecem repetidos em vários trabalhos ${ }^{27-31}$, foram identificados quatro casos deT VP em 2.812 membros tratados com endolaser, o que representa uma incidência de 0,14\%, sem nenhum caso de EP (T abela 8). Se somarmos a essas séries a casuística do presente trabalho, com 417 membros tratados com endolaser sem nenhuma ocorrência tromboembólica, a incidência de TV P com essa técnica será de $0,12 \%$, ou seja, quatro casosem 3.229 membros tratados.

Por outro lado, complicações tromboembólicas também são descritas na cirurgia convencional de varizes. Segundo Keith \& Smead ${ }^{32}$, a incidência de TVP pós-safenectomia é de $0,6 \%$ (três casos em 544 cirurgi-

T abela 6 - Efeitos adversos relatados na literatura

\begin{tabular}{lc}
\hline Efeito adverso & N úmero de membros (\%) \\
\hline D or leve a moderada no trajeto da veia & 80 a $100 \%$ \\
Equimoses & 70 a $100 \%$ \\
Enduração no trajeto da veia tratada & 60 a $100 \%$ \\
Flebite em colaterais não-tratadas & 5 a $10 \%$ \\
H iperpigmentação & 0 a $4 \%$ \\
Insucesso no tratamento & 0 a $7 \%$ \\
Extensão do trombo para a VFC & Três casos relatados \\
Tromboflebite VSM /VSP & N enhum caso \\
Trombose venosa profunda & Q uatro casos relatados \\
Fístula AV iatrogênica & U m caso relatado \\
Parestesia & 0 \\
Embolia pulmonar & 0 \\
Q ueimadura & 0
\end{tabular}

VFC = veia femoral comum; VSM = veia safena magna; VSP = veia safena parva 
as). Lofgren et al. 33 relatam uma incidência de 0,39\% de EP (16 casos em 4.000 cirurgias) pós-safenectomia. $M$ ais recentemente, Bounameaux $\& \mathrm{H}_{\text {uber }}{ }^{34}$ sugeriram que a incidência de EP após a cirurgia de varizes tenha sido de $0,56 \%$.

A percentagem de complicações tromboembólicas relatadas para os dois métodos, até o presente momento, parece não divergir muito a favor de um ou outro procedimento. D essa forma, acreditamos queo EV L seja um método seguro eque não adiciona riscosao paciente, quando comparado com a cirurgia convencional.

A ocorrência de equimoses em um grande número de membros tratados $(60,2 \%)$, bem como de hematoma em dois membros, foi atribuída à infiltração intumescente de um volume considerável de solução fisio-

T abela 7 - T axa de sucesso das séries relatadas na literatura

\begin{tabular}{lcccc}
\hline Estudo & $\begin{array}{c}\text { Compr. onda } \\
\text { laser (nm) }\end{array}$ & $\begin{array}{c}\text { No de membros/ } \\
\text { no de pacientes }\end{array}$ & $\begin{array}{c}\text { Sucesso } \\
\text { imediato }\end{array}$ & $\begin{array}{c}\text { Sucesso } \\
\text { tardio }\end{array}$ \\
\hline $\mathrm{N}$ avarro et al., 20016 & 810 & $40 / 33$ & $100 \%$ & $100 \%$ \\
$\mathrm{M}$ in et al., 200121 & 810 & $90 / 84$ & $97 \%$ & $99 \%$ \\
Chang \& Chua, 2002 & 1.064 & $252 / 149$ & $96,8 \%$ & \\
$\mathrm{M}$ in et al., 200319 & 810 & $499 / 253$ & $98,2 \%$ & $93,4 \%$ \\
Proebstle et al., 200318 & 940 & $109 / 85$ & $96,5 \%$ & $90,4 \%$ \\
Goldman et al., 200428 & 1.320 & $24 / 24$ & $100 \%$ & \\
Proebstle et al., 200529 & 1.320 & $33 / 33$ & $100 \%$ & \\
Agus et al. (IEW G), 2006 30 & $810-980$ & $1.076 / 1.050$ & $100 \%$ & $97 \%$ \\
Viarengo et al., 2006* & 980 & $417 / 253 *$ & $97,1 \%$ & $92,7 \%$ \\
\hline
\end{tabular}

* Série referente ao presente trabalho.

IEWG = Italian Endovenous-Laser Working Group.

Tabela 8 - Complicações tromboembólicas relatadas nas principais séries clínicas de terapia en dovenosa alaser

\begin{tabular}{|c|c|c|c|c|c|}
\hline Estudo & $\begin{array}{c}\text { Comprimento } \\
\text { de onda } \\
\text { do laser }(\mathrm{nm})\end{array}$ & $\begin{array}{l}\mathrm{N} 0 \text { de membros/ } \\
\mathrm{n} \text { - de pacientes }\end{array}$ & $\begin{array}{c}\text { No } \text { de membros } \\
\text { seguidos } \\
\text { com US }\end{array}$ & TVP & EP \\
\hline $\mathrm{N}$ avarro et al., $2001^{6}$ & 810 & $40 / 33$ & & 0 & 0 \\
\hline Gerard et al., 200222 & 980 & $20 / 20$ & & 0 & 0 \\
\hline Chang \& Chua, $2002^{23}$ & 1.064 & $252 / 149$ & & 0 & 0 \\
\hline M in et al., $2003^{19}$ & 810 & $504 / 423$ & & 0 & 0 \\
\hline Proebstle et al., $2003^{18}$ & 940 & $109 / 85$ & & 0 & 0 \\
\hline O h et al., $2003^{24}$ & 980 & $15 / 12$ & & 0 & 0 \\
\hline Perkowski et al., $2004^{25}$ & 940 & $203 / 165$ & & 0 & 0 \\
\hline Timperman, $2004^{20}$ & $810-940$ & $111 / 87$ & & 1 & 0 \\
\hline M ozes et al., $2005^{27}$ & 810 & $56 / 41$ & & 3 & 0 \\
\hline Goldman et al., $2004^{28}$ & 1.320 & $24 / 24$ & & 0 & 0 \\
\hline Lisbona et al., 200431 & 810 & $369 / 295$ & & 0 & 0 \\
\hline Viarengo et al., 2006* & 980 & $417 / 253$ & & 0 & 0 \\
\hline Proebstle et al., $2005^{29}$ & 1320 & $33 / 33$ & & 0 & 0 \\
\hline Agus et al. (IEW G ), $2006^{30}$ & $810-980$ & $1.076 / 1.050$ & & 0 & 0 \\
\hline
\end{tabular}

* Série referente ao presente trabalho.

Artigos com relatos que incluíam as mesmas séries foram excluídos.

US = ultra-som; TVP = trombose venosa profunda; EP = embolia pulmonar; IEWG = Italian Endovenous-Laser Working Group. 
lógica com lidocaína sem vasoconstrictor ao longo do trajeto venoso, etambém à perfuração da parede venosa pelo impacto direto do laser, por contato com a fibra propriamente dita.

Podemos afirmar que o método é clinicamente exeqüível em regime ambulatorial, com anestesia estritamente local, sendo uma técnica bem tolerada e aceita pelos pacientes, podendo, inclusive, ser realizada em pacientes com úlcera ativa. $\mathrm{N}$ esses casos, fica como um método de indicação específica para aqueles pacientes com grave fibrose no terço distal das pernas ou úlcera em atividade, situações em que a cirurgia convencional apresenta sérias limitações técnicas. Além disso, esse método oferece uma rara oportunidade para se avaliar o papel da ligadura da JSF e tributárias da crossa no controle da recidiva de varizes, pois permite obliterar efetivamente a VSM, sem a necessidade de abordar cirurgicamente a crossa da V SM . Entretanto, é fundamental que o método seja realizado sob estrito controle com eco-D oppler. Consideramos esse controle fundamental para o sucesso do tratamento e segurança do paciente. Basear-se na orientação da luz-guia, por transiluminação, não é seguro, sobretudo em pacientes magros ecom a crossa da V SM retificada, situações em que a fibra penetra facilmente no sistema venoso profundo, sem que ocorram alterações significativas e facilmente perceptíveis nos níveis de luminosidade por transiluminação. Além disso, os baixosíndices de complicações dependem, em parte, de uma adequada e efetiva infiltração intumescente perivasal, envolvendo toda a circunferência do vaso, e isso só é possível sob orientação com eco-D oppler.

A eficácia do endolaser na obtenção de oclusão imediata da VSM na ordem de $97 \%$ é muito satisfatória, sendo um motivo razoável para a avaliação desse novo método.

\section{C onclusão}

0 tratamento endovenoso devarizescom laser éum método eficaz para oclusão da VSM , com recorrência inferior a 8\% no período de seguimento descrito, e os ef eitos adversos observados foram autolimitados.

\section{Referências}

1. M affei $F H, M$ agaldi $C$, Pinho SZ, et al. Varicose veins and chronic venous insufficiency in Brazil: prevalence among 1755 inhabitants of a country town. Int J Epidemiol. 1986;15:210-7.
2. Bergan J, Ballard JI. Correction of superficial reflux. In: G loviczki P, Bergan JJ, editors. Atlas of endoscopic perforator vein surgery. London: Springer; 1998. p 98-103.

3. L ofgren $E P, L$ ofgren $K A$. R ecurrence of varicoseveinsafter the stripping operation. Arch Surg. 1971;102:111-4.

4. D odd H , C ockett FB. The pathology and surgery of the veins of the lewer limb. 2nd ed. London: Livingstone; 1976. p. 210-4.

5. D avies AH, Steffen C, Cosgrove C, W ilkins DC. V aricose vein surgery: patient satisfaction. J R Coll Surg Edinb. 1995;40:298-9.

6. N avarro L, M in RJ, Bone C. Endovenous Laser: a new minimally invasive method of treatment of varicose veinspreliminary observationsusing an $810 \mathrm{~nm}$ diodelaser. D ermatol Surg. 2001;27:117-22.

7. ProebstleTM, Lehr H A, K argl A, et al. Endovenoustreatment of the greater saphenous vein with a 940-nm diode laser: thrombotic occlusion after endoluminal thermal damage by laser- generated steam bubbles. J V asc Surg. 2002;35:729-36.

8. Timperman PE, Sichlau M, Ryu RK. G reater energy delivery improves treatment success of endovenous laser treatment of incompetent saphenous veins. J V asc Interv Radiol. 2004;15:1061-3.

9. D werryhouseS, D aviesB, H arradineK, Earnshaw JJ. Stripping the long saphenous vein reduces the rate of reoperation for recurrent varicoseveins: five-year results of arandomized trial. J V asc Surg. 1999;29:589-92.

10. M anfrini S, G asbarro V, D anielsson G, et al. Endovenous management of saphenous vein reflux. Endovenous R eflux M anagement Study G roup. J V asc Surg. 2000; 32:330-42.

11. Glass GM. N eovascularisation in recurrence of the varicose great saphenous vein following transection. Phlebology. 1987;2:81-92.

12. N yamekyel, Shephard N A, D avies B, H eather BP, Earnshaw J). Clinicopathological evidence that neovascularisation is a cause of recurrent varicose veins. Eur J V asc Endovasc Surg. 1998;15:412-5.

13. van Rij $A M$, J ones $G T$, H ill GB, Jiang P. N eovascularization and recurrent varicose veins: more histologic and ultrasound evidence. J Vasc Surg. 2004;40:296-302.

14. Fischer R, LindeN , D uff C, J eanneret C, C handler J G, Seeber $P$. L aterecurrent saphen ofemoral junction reflux after ligation and stripping of the greater saphenous vein. J V asc Surg. 2001;34:236-40.

15. C reton D. Prostheticmaterial interposition on thecrossectomy stump in varicose vein recurrence surgery: preliminary report on theprevention of angiogenesis. ScriptaPhlebol. 1998;6:4-7.

16. G lassG M . Prevention of saphen ofemoral and saphenopopliteal recurrence of varicose veins by forming a partition to contain neovascularisation. Phebology. 1998;13:3-9.

17. Jakobsen $\mathrm{BH}$. The value of different forms of treatment for varicose veins. Br J Surg. 1979;66:182-4.

18. ProebstleTM , Gul D, Lehr H A, Kargl A, K nop J. Infrequent early recanalization of greater saphenousvein after endovenous laser treatment. J V as Surg. 2003;38:511-6.

19. $M$ in RJ, K hilnani $N$, Z immet SE. Endovenouslaser treatment of saphenous vein reflux: long-term results. J V asc Interv Radiol. 2003;14:991-6. 
20. T imperman PE. Arteriovenous fistula after endovenous laser treatment of the short saphenous vein. J V asc Interv Radiol. 2004;15:625-7.

21. $M$ in RJ , Z immet SE, I saacs M N , Forrestal M D. Endovenous laser treatment of the incompetent greater saphenous vein. J V asc Interv Radiol. 2001;12:1167-71.

22. G erard JL, D esgranges $P, B$ ecquemin JP, D esse H, M elliere $D$. Peut-on traiter les grandes saphénes variqueuses par laser endoveineaux en ambulatoire. J M al V asc. 2002;27:222-5.

23. Chang CJ, Chua JJ. Endovenous laser photocoagulation (EV LP) for varicose veins. LasersSurg M ed. 2002;31:257-62.

24. O h CK, Jung DS, Jang HS, Kwon KS. Endovenous laser surgery of theincompetent greater saphenous vein with a 980 $\mathrm{nm}$ diode laser. Dermatol Surg. 2003;29:1135-40.

25. Perkowski P, Ravi R, Gowda RC, et al. Endovenous laser ablation of the saphenous vein for treatment of venous insufficiency and varicose veins: early results from a large single-center experience. J Endovasc Ther. 2004;11:132-8.

26. Soracco JE, D Ámbola JL, Ciucci JL, Godoy JM P, Belczak CEQ. Complicações no tratamento com laser endovascular em varizes demembros inferiores. J V asc Bras. 2005;4:333-5.

27. Mozes G, Kalra M, Carmo M, Swenson L, Gloviczki P. Extension of saphenous thrombus into the femoral vein: a potential complication of new endovenousablation techniques. J V asc Surg. 2005;41:130-5.

28. Goldman M P, M auricio M, Rao J. Intravascular 1320-nm laser closure of the great saphenous vein: a 6- to 12-month follow-up study. Dermatol Surg. 2004;30:1380-5.

29. ProebstleT M , M oehler T, G ül D , H erdemann S. Endovenous treatment of the great saphenous vein using a 1,320 nm $\mathrm{N}$ d:YAG laser causes fewer side effects than using a $940 \mathrm{~nm}$ diode laser. Dermatol Surg. 2005;31:1678-83; discussion 1683-4.
30. Agus G B, M ancini S, M agi G, IEW G. The first 1000 cases of I talian Endovenous-Laser W orking G roup (IEW G). R ationale, and long-term outcomesfor the1999-2003 period. Int Angiol. 2006;25:209-15.

31. Lisbona $C$, Pérez $P$, Segura J, Puncernau $X$. Laser surgery in venous insufficiency: E.L.A.V. personal technique. J Vasc Bras. 2004;3:403-6.

32. Keith LM Jr., Smead WL. Saphenous vein stripping and its complications. Surg Clin N orth Am. 1983;63:1303-12.

33. Lofgren EP, Coates $H L, O$ 'Brien PC. Clinically suspect pulmonary embolism after vein stripping. $M$ ayo $C$ lin Proc. 1976;51:77-80.

34. BounameauxH, $\mathrm{H}$ uber $\mathrm{O}$. Postoperative deep vein thrombosis and surgery for varicose veins. BM J. 1996;312:1158.

Correspondência:

Luiz M arcelo Aiello Viarengo

Rua José Aiello, 23

CEP 13206-551 - Jundiaí, SP

Tel./Fax: (11) 4586.4444

E-mail: viarengo@terra.com.br

O conteúdo do J Vasc Bras está disponível em português e em inglês

no site do Jornal Vascular Brasileiro em

www.jvascbr.com.br 